\title{
Experimental limits for Eigenvalue Calibration in Liquid-Crystal Mueller-Matrix Polarimeters
}

\author{
Neil C. Bruce ${ }^{1 *}$, Oscar G. Rodríguez-Herrera ${ }^{1}$, Juan Manuel López- \\ TÉLlez ${ }^{2}$, OMAR ROdRÍGUEZ-NUÑEZ ${ }^{1}$ \\ ${ }^{1}$ Centro de Ciencias Aplicadasy Desarrollo Tecnológico, Universidad Nacional Autónoma de México, Circuito Exterior, Ciudad Universitaria, \\ Ciudad de México 04510, México \\ ${ }^{2}$ College of Optical Sciences, University of Arizona, 1630 E. University Blvd., Tucson Az, 85721 USA \\ *Corresponding author: neil.bruce@ccadet.unam.mx
}

Received XX Month XXXX; revisedXX Month, XXXX; accepted XX MonthXXXX; posted XX Month XXXX (Doc. ID XXXXX); published XX Month XXXX

\begin{abstract}
A numerical study is carried out to find the experimental conditions necessary for the eigen-value calibration procedure to work correctly in a liquid-crystal variable retarder based Mueller-matrix polarimeter. Using the error between the simulated experimental Mueller matrix in a polarimeter with errors and the expected ideal Mueller matrices for 4 calibration samples, the maximum experimental errors are estimated for a succesful eigen-value calibration. It is found that the retarder axes orientations have smaller permitted errors than the retardation values. (c) 2018 Optical Society of America
\end{abstract}

OCIS codes: (120.5410) Polarimetry, (260.5430) Polarization, (230.5440) Polarization-selective devices

http://dx.doi.org/10.1364/OL.99.099999

Optical Mueller-matrixpolarimetry techniques have advanced rapidly in recent years[1-11]. In particular, optimized polarimeters are now designed and implemented, and calibration techniques are used to reduce the effects of systematic errors and random noise in the final Mueller matrix. In this paper we study the limits of applicability of the eigenvalue calibration procedure introduced by Compain and coworkers[12-14]. This method has been found to be a very robust and reliable calibration procedure $[13,14]$, although many different calibration methods have been proposed (for example [15] and the references therein). Obviously, if the original measured data is very noisy, no calibration procedure will work correctly, so here we find the limits of systematic experimental errors in the polarimeter, and the random noise in the intensity measurements for which the eigenvalue calibration procedure may not work.

A typical experimental setup for a Mueller-matrix polarimeter using liquid-crystal variable retarders (LCVRs) is shown in Figure 1. The important parameters in the polarimeter are the four retardance values of the LCVRs $\left(\delta_{1}, \delta_{2}, \delta_{3}, \delta_{4}\right)$, and the directions of the retarder axes $\left(\theta_{1}, \theta_{2}, \theta_{3}, \theta_{4}\right)$, where the sub-index refers to the retarder number. Varying the parameters of the polarimeter, the Stokes vectors incident on the sample, and detected leaving the sample can be varied. To calculate the 16 elements of the Mueller matrix at least 16 combinations of the incident and detected Stokes vector are required, and this can be achieved by using a combination of four incident and four detected Stokes vectors. In this case, the matrix formed by the four incident Stokes vectors is the characteristic matrix $C^{P S G}$ of the Polarization State Generator (PSG, formed by $P_{1}, R_{1}$ and $R_{2}$ in Figure 1 ) and the matrix formed by the four detected Stokes vectors is the characteristic matrix $C^{P S A}$ of the Polarization State Analyzer (PSA, formed by $R_{3}, R_{4}$ and $P_{2}$ in Figure 1) [2]:
$C^{P S G}=\left(\begin{array}{llll}S^{\text {inc1 }} & S^{\text {inc2 }} & \cdots & S^{\text {incN }}\end{array}\right)$
$C^{P S A}=\left(\begin{array}{llll}S^{\text {det1 }} & S^{\text {det2 }} & \cdots & S^{\text {detN }}\end{array}\right)$

where $\mathrm{N}$ is the number of different Stokes vectors used in the PSG and the PSA. Tyo [11] showed that it is possible to optimize a Mueller-matrix polarimeter by making the condition numbers (the ratio of the largest singular value divided by the smallest singular value), of the characteristic matrices of the PSG and the PSA as small as possible. Tyo showed that, for the reconstruction of the Mueller matrix, the minimum condition number is equal to $\sqrt{3}=1.7321$ for $C^{P S G}$ and $C^{P S A}$.

Finding the minimum condition numbers for the many different possible configurations of a polarimeter gives many possible parameter combinations, but one solution, found and used by DeMartino and coworkers [6], and Smith and coworkers [7] is the set of parameters:

$\theta_{1}=\theta_{4}=27.4^{\circ}$

$\theta_{2}=\theta_{3}=72.4^{\circ}$

where the subindex indicates which retarder the axis position refers to, and the retardance values used in the PSG and the PSA are given by the following sequence with $\Delta_{1}=135^{\circ}$ and $\Delta_{2}=315^{\circ}$ : 


$$
\begin{aligned}
& \left(\delta_{1}, \delta_{2}, \delta_{3}, \delta_{4}\right)=\left(\Delta_{1}, \Delta_{1}, \Delta_{1}, \Delta_{1}\right),\left(\Delta_{1}, \Delta_{1}, \Delta_{1}, \Delta_{2}\right) \\
& \left(\Delta_{1}, \Delta_{1}, \Delta_{2}, \Delta_{1}\right),\left(\Delta_{1}, \Delta_{1}, \Delta_{2}, \Delta_{2}\right),\left(\Delta_{1}, \Delta_{2}, \Delta_{1}, \Delta_{1}\right) \\
& \left(\Delta_{1}, \Delta_{2}, \Delta_{1}, \Delta_{2}\right),\left(\Delta_{1}, \Delta_{2}, \Delta_{2}, \Delta_{1}\right),\left(\Delta_{1}, \Delta_{2}, \Delta_{2}, \Delta_{2}\right) \\
& \left(\Delta_{2}, \Delta_{1}, \Delta_{1}, \Delta_{1}\right),\left(\Delta_{2}, \Delta_{1}, \Delta_{1}, \Delta_{2}\right),\left(\Delta_{2}, \Delta_{1}, \Delta_{2}, \Delta_{1}\right) \\
& \left(\Delta_{2}, \Delta_{1}, \Delta_{2}, \Delta_{2}\right),\left(\Delta_{2}, \Delta_{2}, \Delta_{1}, \Delta_{1}\right),\left(\Delta_{2}, \Delta_{2}, \Delta_{1}, \Delta_{2}\right) \\
& \left(\Delta_{2}, \Delta_{2}, \Delta_{2}, \Delta_{1}\right),\left(\Delta_{2}, \Delta_{2}, \Delta_{2}, \Delta_{2}\right)
\end{aligned}
$$

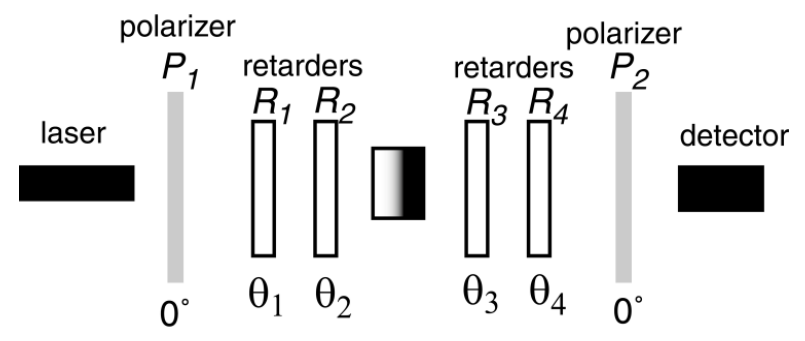

Figure 1: Experimental setup for a Mueller-matrix polarimeter. The angles associated with each component refer to the relative angle of the optical axis of that component with respect to the horizontal plane.

In this paper, we perform a numerical simulation of the polarimeter considering an experimental setup that can have systematic errors in the experimental polarimeter parameters. It is assumed that the errors in the PSG and PSA are the same so that both sections of the polarimeter have the same condition number. These errors mean that the condition numbers of the experimental polarimeter are not optimized, and we can study the behavior of the system as the systematic experimental errors change. We also include random errors in the measurement of the experimental intensities; these errors vary from a minimum of $0.5 \%$ to a maximum of $5 \%$ of Additive White Gaussian Noise. The experimental intensities are simulated for the four calibration samples suggested by de Martino et al. (air (no sample), horizontal polarizer, vertical polarizer and quarter-wave retarder with its fast axis at $30^{\circ}$ to the horizontal) [6], for the Compain et al. eigenvalue calibration process [12]. Note that in practice the Mueller matrices of the measured calibration samples can be averaged over various matrices to reduce the noise. This was not done in our simulation. The Mueller matrices of these samples are calculated from the intensity measurements using the method described by Chipman [2], assuming an ideal polarimeter without errors. Finally, the eigenvalue calibration procedure introduced by Compain and coworkers is used to reduce the errors in the final Mueller matrices. The Compain method finds the experimental Mueller matrices describing the polarimeter through a mapping of the experimental and theoretical Mueller matrices for these calibration samples. The polarimeter system matrices are then found by finding the eigenvector associated with the smallest eigenvalue of this mapping [12]. In the results presented in this paper we used a range of $\pm 20^{\circ}$ in each of the parameters of the polarimeter (two axes angles and two retardance values), with a step of $2^{\circ}$, giving a total of $21^{4}=194481$ sample points for each case studied. The equations were programmed in MatLab and took approximately 2 hours to run for each value of the measurement error on a computer working at $3.4 \mathrm{GHz}$.
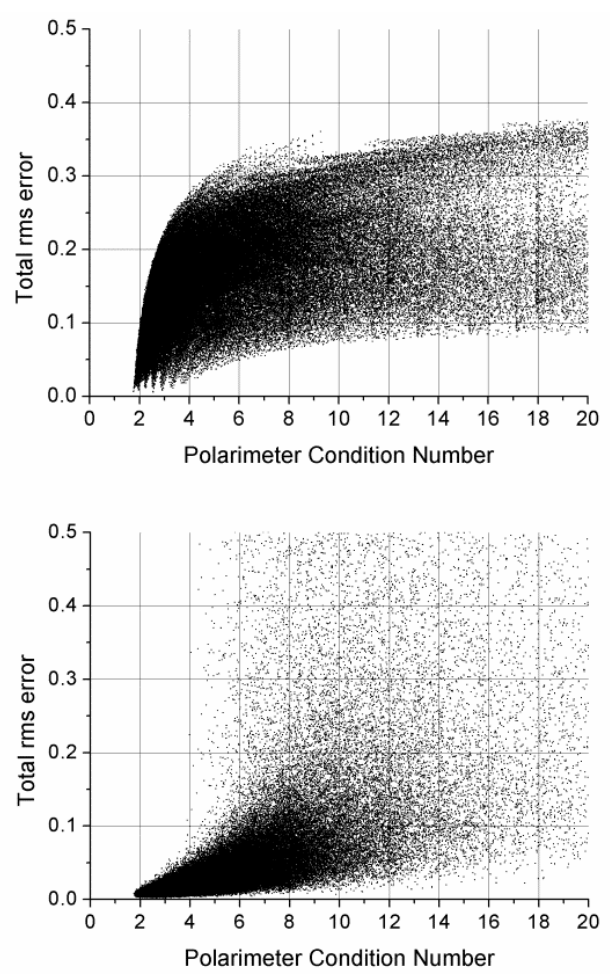

Figure 2: The total rms error for the case of 1.5\% measurement error, before the eigenvalue calibration (top) and after the eigenvalue calibration (bottom), versus the condition number of the polarimeter.

The errors in the Mueller matrices calculated in the simulation were found using the total rms difference between the known, correct calibration sample Mueller matrices, and the calculated matrices, both before and after the calibration procedure, to find the effect of this process. The total rms error is calculated using:

$$
r m s=\frac{1}{64} \sqrt{\sum_{N=1}^{4} \sum_{i=1}^{4} \sum_{j=1}^{4}\left(M_{i j N}^{S}-M_{i j N}^{\text {theorys }}\right)^{2}}
$$

where $M_{i j}^{S}$ are the $i, j$ components of the simulated Mueller matrix including the errors in the polarimeter parameters and the noise in the measured intensity, and $M_{i j}^{\text {theorys }}$ are the $i, j$ components of the theoretically expected Mueller matrix for the known calibration sample. The subindex $N$ in this case indicates the matrices for each of the four calibration samples.

Figure 2 shows an example of the total rms error values as a function of the condition number of the polarimeter, both before and after the calibration process, for a random measurement error of $1.5 \%$. Each point represents the total rms error for a particular configuration of the experimental polarimeter parameters. It can be seen that in both cases, as the condition number increases the polarimeter becomes more unstable with larger total rms errors, and also that the calibration process reduces the spread of the total rms error for low condition numbers but increases the spread for larger condition numbers. In fact, for larger condition numbers the calibration algorithm can fail, and give no solution, for some cases. 

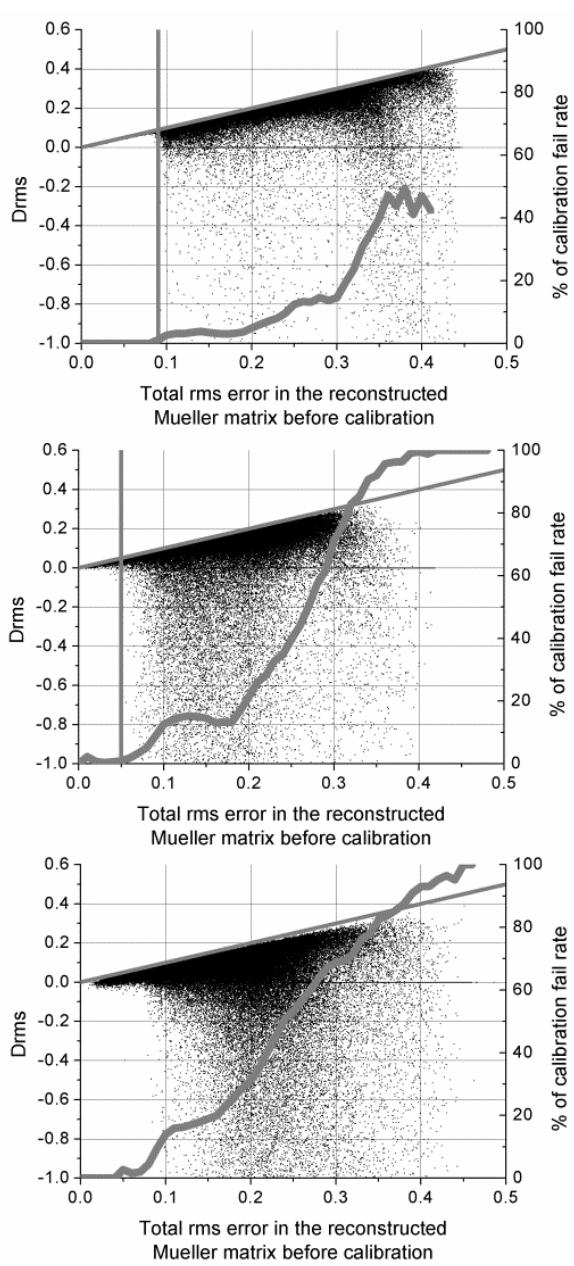

Figure 3: The difference in rms error before and after the eigenvalue calibration for a measurement error of $0.5 \%$ (top), $1.5 \%$ (middle) and $5 \%$ (bottom). The diagonal gray lines mark lines of slope 1 (perfect correction by the calibration), and the vertical lines mark the estimated limits of good correction by the calibration method. The thick gray curves show the percentage (right y-axis) of the cases for which the calibration fails for each value of the rms error before the calibration.

The effect of the calibration procedure can be taken into account by calculating the difference between the total rms error before and after the calibration for each polarimeter configuration:

Drms $=r m s_{\text {before }}-r m s_{\text {after }}$

If this difference is zero then the calibration has no effect, if it is positive, then the calibration has reduced the error in the final results, and if it is negative, then the calibration has increased the error and has not worked.

In Figure 3 we present the results of the difference parameter given by equation (7) as a function of the total rms error in the uncalibrated results, for the cases of a random error in the measurement of the detected intensity of $0.5 \%, 1.5 \%$ and $5 \%$. It can be seen that for a random measurement error of $0.5 \%$ and for small errors in the uncalibrated data, the eigenvalue calibration procedure corrects the results completely (the points lie on the line of slope 1 in the graph). For a measurement error of $5 \%$, it can be seen that the data points are slightly separated from the line of slope 1, indicating that no data is perfectly corrected for this case. In general, as the random measurement error increases or as the total rms error increases in the uncalibrated data, the calibration results become more unstable. The concentration of points on a horizontal line for a value of the difference parameter equal to zero corresponds to the cases when the calibration algorithm used fails and gives no solution. It can also be seen that, as the random measurement error increases, the percentage of cases for which the calibration method fails, increases more rapidly.
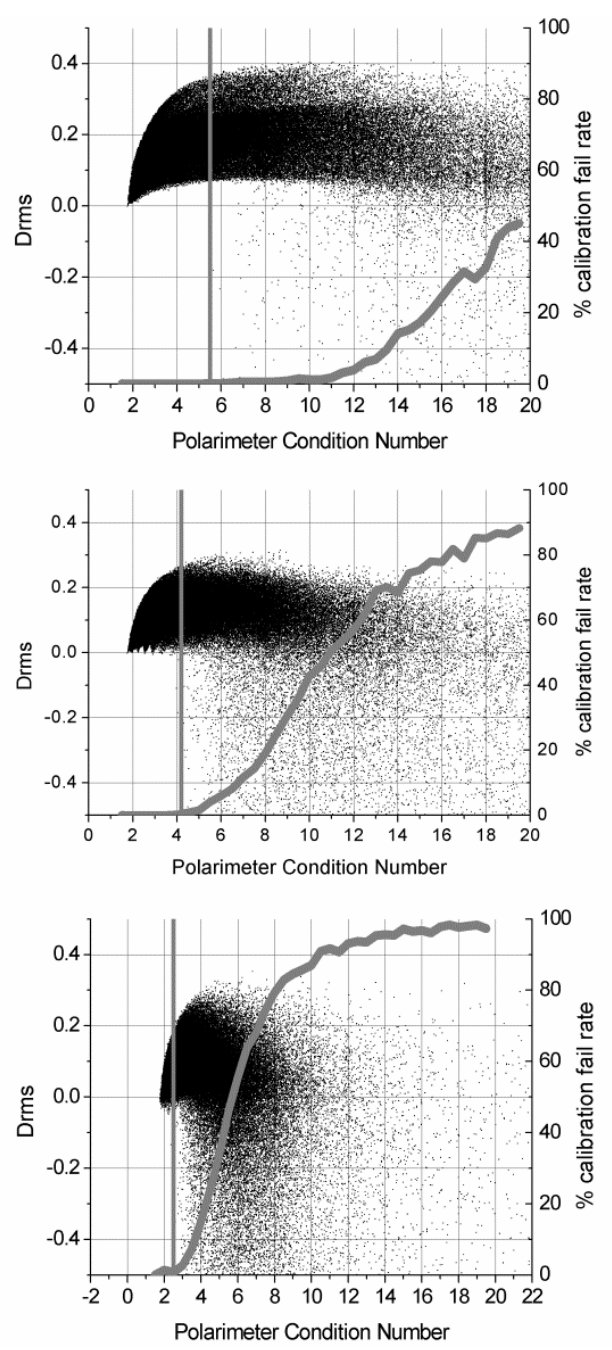

Figure 4: The difference in rms error before and after the eigenvalue calibration for a measurement error of $0.5 \%$ (top), $1.5 \%$ (middle) and

$5 \%$ (bottom). The vertical lines mark the estimated limits of good correction by the calibration method. The thick gray curves show the percentage (righty-axis) of the cases for which the calibration fails.

In Figure 4 we graph the same data, but now as a function of the polarimeter condition number, which is more directly related to the errors in the experimental polarimeter configuration, for the same random measurement errors as in Figure 3. Again, as the condition number increases or as the random measurement error increases, the calibration procedure becomes more unstable.

One aspect that is particularly interesting in Figures 3 and 4 is that for the measurement errors of $5 \%$, the calibration procedure is unstable for all the results presented here, except for the optimized polarimeter, with no errors (condition number equal to $\sqrt{3}$ for the 
PSG and the PSA). It can be seen that there are rms difference values (equation 7) that are negative for almost the full range of total rms error in the uncalibrated data, and for almost the full range of polarimeter condition number. Whether the calibration works or not depends on the particular polarimeter configuration.

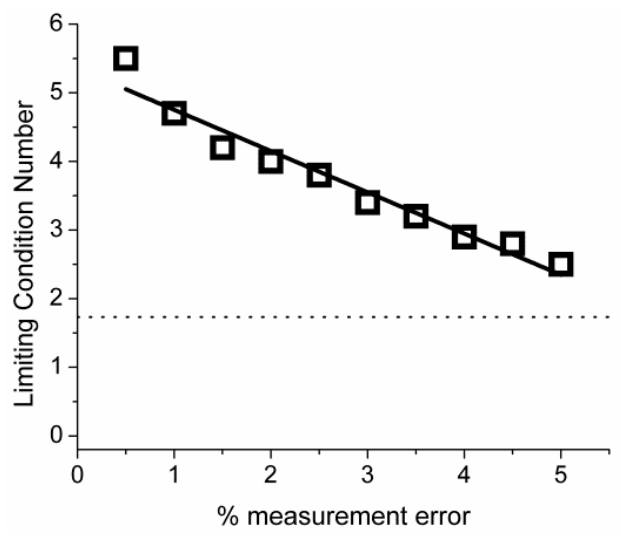

Figure 5: The variation of the limiting condition number of the polarimeter for varying levels of measurement error. The open squares are the numerical results, the solid line is a linear fit to this data, and the dotted horizontal line is the ideal condition number.

Figure 5 shows the estimated limiting value of the condition number for which the calibration process always works for the different measurement errors considered. This is obtained from the graphs of Figure 4 by estimating the condition number value at which the negative values of Drms become more than $0 \%$ of the total number of cases, and the values are shown in figure 4 by the vertical gray lines. It can be seen that, as expected, the polarimeter has to be closer to optimized for larger random measurement errors. The linear fit to the numerical data gives the equation:

LimitCondNum $=(-0.60 \pm 0.05) R a n E r r+(5.35 \pm 0.15)$

with an $R^{2}$ value of 0.95 . Of course, for smaller values of the random measurement error, the limiting values of the condition number tend to be much higher than this linear fit suggests (in fact, with perfect measurement, or $0 \%$ random measurement error, the calibration process always works), but equation (8) and Figure 5 give an estimation of how well the polarimeter has to be aligned for many practical situations.

Finally, Figure 6 shows the permitted values of the errors in the polarimeter configuration to obtain the limiting condition numbers in Figure 5 . These values were obtained by analyzing the data for all of the polarimeter configurations in the simulation with condition number between the minimum value and the desired limiting condition number. The values plotted in the figure are the standard deviations of a Gaussian fit to the parameter values for each condition number. From the data, it can be seen that it is more important to control the retarder axes than the retardance values to ensure a good final result. It can also be seen that the permitted errors are rather large, of the order of a few degrees or more, depending on the polarimeter parameter, which indicates the stability and robustness of the Compain calibration procedure. It is also interesting that the results shown here indicate that there are limits on the eigenvalue calibration method, and support the need for alternative calibration methods [15]. It is important to point out that the simulation performed in this paper is limited in the sense that the errors in the experimental setup are assumed to be systematic only, and there is no random component. Also the pairs of retardances and retarder axes angles have the same error, which probably would not occur in practice; however the results found here give a first approximation to the behaviour of real systems and show the limits of the eigenvalue calibration method.

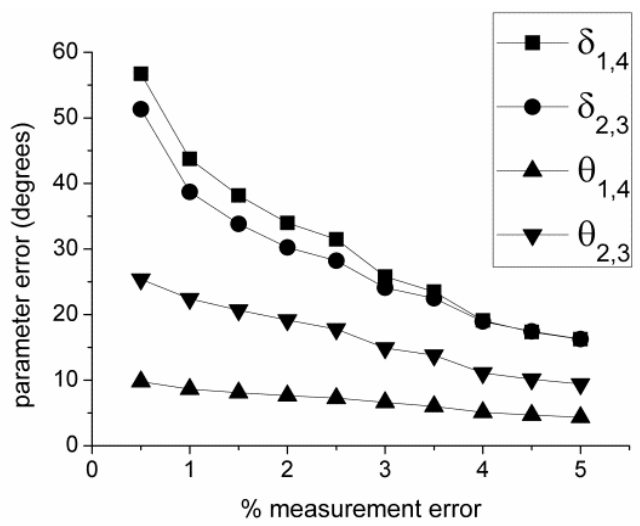

Figure 6: The variation of the standard deviations of the parameter errors for the polarimeter for varying levels of measurement error.

Funding. DGAPA-UNAM (PAPIIT IT100114 and IT100417).

Acknowledgments. Omar Rodríguez Nuñez acknowledges a doctoral grant and Juan Manuel López Téllez acknowledges a postdoctoral research grant, both from CONACyT, Mexico.

\section{References}

1. D. Goldstein, Polarized Light, Second Edition, CRC Press, Boca Raton, (2003)

2. R.A. Chipman, Polarimetry, in Handbook of Optics, M. Bass editor, McGraw-Hill, Columbus, (1995), Chapter 22

3. D.H. Goldstein, Applied Optics, 31, (1992), 6676-6683

4. G. Martínez-Ponce, C. Solano and C. Pérez.Barrios, Optics and Lasers in Engineering, 49, (2011), 723-728

5. J.M. Bueno and P. Artal, Optics Letters, 24(1), (1999), 64-66

6. A. de Martino, Y-K- Kim, E. García-Caurel, B. Laude and B. Drévillon, Optics Letters, 28(8), (2003), 616-618

7. M.H. Smith, Applied Optics, 41(13), (2002), 2488-2493

8. R.C. Thompson, J.R. Bottiger and E.S. Fry, Applied Optics, 19, (1980), 13231332

9. J. M. López-Téllez and N. C. Bruce, Applied Optics, 53(24), (2014), 53595366

10. W.S. Bickel and W.M. Bailey, Stokes vectors, American Journal of Physics, 53, (1985), 468-478

11. J.S. Tyo, Applied Optics, 41(4), (2002), 619-630

12. E. Compain, S. Poirier and B. Drevillon, Applied Optics, 38(16), (1999), 3490-3502

13. D. Lara-Saucedo, PhD thesis, Imperial College, London, (2005)

14. O.G. Rodríguez-Herrera, PhD thesis, National University of Ireland, Galway, (2009)

15. B.G. Hoover, D.A. Rugely, C.M. Francis, G. Zeira, V.L. Gamiz, Optics Express, 24(17), (2016), 19881-19893 
1. D. Goldstein, Polarized Light, Second Edition, CRC Press, Boca Raton, (2003)

2. R.A. Chipman, Polarimetry, in Handbook of Optics, M. Bass editor, McGraw-Hill, Columbus, (1995), Chapter 22

3. D.H. Goldstein, Mueller matrix dual-rotating-retarder polarimeter, Applied Optics, 31, (1992), 6676-6683

4. G. Martínez-Ponce, C. Solano and C. Pérez.Barrios, Hybrid complete Mueller polarimeter based on phase modulators, Optics and Lasers in Engineering, 49, (2011), 723-728

5. J.M. Bueno and P. Artal, Double-pass imaging polarimetry in the human eye, Optics Letters, 24(1), (1999), 64-66

6. de Martino, Y-K- Kim, E. García-Caurel, B. Laude and B. Drévillon, Optimized Mueller polarimeter with liquid crystals, Optics Letters, 28(8), (2003), 616-618

7. M.H. Smith, Optimization of a dual-rotating-retarder Mueller matrix polarimeter, Applied Optics, 41(13), (2002), 2488-2493

8. R.C. Thompson, J.R. Bottiger and E.S. Fry, "Measurement of polarized interactions via the Mueller matrix", Applied Optics, 19, (1980), 13231332

9. J. M. López-Téllez and N. C. Bruce, “Mueller-matrix polarimeter using analysis of the nonlinear voltage-retardance relationship for liquidcrystal variable retarders", Applied Optics, 53(24), (2014), 5359-5366

10. W.S. Bickel and W.M. Bailey, Stokes vectors, Mueller matrices and polarized scattered light, American Journal of Physics, 53, (1985), 468478

11. J.S. Tyo, Design of optimal polarimeters: maximization of signal to noise ratio and minimization of systematic errors, Applied Optics, 41(4), (2002), 619-630

12. E. Compain, S. Poirier and B. Drevillon, General and self-consistent method for the calibration of polarization modulators, polarimeters and Mueller-matrix ellipsometers, Applied Optics, 38(16), (1999), 34903502

13. D. Lara-Saucedo, Three-dimensional complete polarisation sensitive imaging using a confocal Mueller matrix polarimeter, $\mathrm{PhD}$ thesis, Imperial College, London, (2005)

14. O.G. Rodríguez-Herrera, Far-field method for the characterization of three-dimensional fields: vector polarimetry, PhD thesis, National University of Ireland, Galway, (2009)

15. B.G. Hoover, D.A. Rugely, C.M. Francis, G. Zeira, V.L. Gamiz, Bistatic laser polarimeter calibrated to $1 \%$ at visible-SWIR wavelengths, Optics Express, 24(17), (2016), 19881-19893 